\title{
The impact of ECG synchronization during acquisition of left-atrium computed tomography model on radiation dose and arrhythmia recurrence rate after catheter ablation of atrial fibrillation - a prospective, randomized study
}

\author{
Skala $\mathrm{T}^{1}$, Tudos $\mathrm{Z}^{2}$, Homola $\mathrm{M}^{3}$, Moravec $\mathrm{O}^{1}$, Kocher $\mathrm{M}^{2}$, Cerna $\mathrm{M}^{2}$, Ctvrtlik $\mathrm{F}^{2}$, Odstrcil $\mathrm{F}^{4}$, \\ Langova $\mathrm{K}^{5}$, Klementova $\mathrm{O}^{6}$, Taborsky $\mathrm{M}^{1}$
}

Department of Internal Medicine I - Cardiology, Faculty of Medicine and Dentistry, Palacky University

Olomouc and University Hospital Olomouc, Czech Republic. Zbynek.Tudos@seznam.cz

\begin{abstract}
BACKGROUND: The impact of ECG gating during computed tomography (CT) acquisition of left atrium (LA) model on radiation dose, image quality and ablation event-free survival rate after catheter ablation (CA) of atrial fibrillation (AF) is not well defined.

METHODS: Sixty-two patients with paroxysmal atrial fibrillation were randomized for two types of LA CT (with vs without ECG gating) before CA. Pulmonary veins isolation was performed in all patients. Patients were followed for 12 months after CA.

RESULTS: There was no difference between the groups in CA length (131.61 $\pm 32.57 \mathrm{vs} 119.84 \pm 33.18 \mathrm{~min} ; \mathrm{p}=0.108)$, CA fluoroscopy time (4.48 \pm 2.19 vs $3.89 \pm 1.83 \mathrm{~min} ; \mathrm{p}=0.251)$, CA fluoroscopy dose (3.99 \pm 2.79 vs $3.91 \mathrm{vs} 2.91 \mathrm{~Gy}{ }^{*} \mathrm{~cm} 2$; $p=0.735)$, visual data quality $(1.77 \pm 0.88$ vs $2.0 \pm 0.63 ; p=0.102)$ and registration error $(2.42 \pm 0.72$ vs $2.43 \pm 0.46 \mathrm{~mm}$; $\mathrm{p}=0.612$ ). We found a significant difference in CT Dose index ( $89.55 \pm 5.99$ vs $19.19 \pm 4.33 \mathrm{mGy} ; \mathrm{p}<0.0001)$ and Dose Length product $\left(1438.87 \pm 147.75\right.$ vs $\left.328.21 \pm 73.83 \mathrm{mGy}{ }^{*} \mathrm{~cm} ; \mathrm{p}<0.0001\right)$. Twelve months after $\mathrm{CA}, 25$ of 31 patients in the gated group and 24 of 31 patients in the non-gated group were free of AF (80.65 vs $77.42 \% ; p=0.838)$. CONCLUSION: ECG gating of computed tomography of LA before AF ablation burdens patients with a four times higher radiation dose while improving neither the quality of CT model or fusion of CT with the electroanatomic map. As a result, it has no significant impact on arrhythmia recurrence rate after ablation (Tab. 3, Fig. 3, Ref. 25). Text in PDF www.elis.sk.

KEY WORDS: atrial fibrillation, multi-detector computed tomography, catheter ablation, ECG gating, radiation dose.
\end{abstract}

\begin{abstract}
Abreviations: AF - atrial fibrillation; CA - catheter ablation; CTDIvol - volume computed tomography dose index; DLP - dose length product; ED - effective dose; FAM - fast electro-anatomic map; LA - left atrium; MDCT - multi-detector computed tomography; PV - pulmonary vein.
\end{abstract}

${ }^{1}$ Department of Internal Medicine I - Cardiology, Faculty of Medicine and Dentistry, Palacky University Olomouc and University Hospital Olomouc, Czech Republic, ${ }^{2}$ Department of Radiology, Faculty of Medicine and Dentistry, Palacky University Olomouc and University Hospital Olomouc, Czech Republic, ${ }^{3}$ Department of Medical Physics and Radiation Protection, University Hospital Olomouc, Czech Republic, ${ }^{4}$ Department of Radiological Methods, Faculty of Health Sciences, Palacky University Olomouc, Czech Republic, ${ }^{5}$ Department of Medical Biophysics, Faculty of Medicine and Dentistry, Palacky University Olomouc, Czech Republic, and ${ }^{6}$ Department of Anesthesiology and Intensive Care Medicine, Faculty of Medicine and Dentistry, Palacky University Olomouc and University Hospital Olomouc, Czech Republic

Address for correspondence: Z. Tüdös, MD, PhD, Department of Radiology, University Hospital, I.P. Pavlova 6, CZ-77900 Olomouc, Czech Republic. Phone: +420.588.445995, Fax: +420.588.442508

Acknowledgement: Study was supported by Palacky University grant IGA_LF_2018_002.

\section{Introduction}

Atrial fibrillation (AF) is the most common type of cardiac arrhythmia. Catheter ablation (CA) is an effective treatment for AF. The cornerstone of CA is pulmonary vein (PVs) isolation. A non-invasive visualization of PVs and left atrium (LA) is used to assess the LA anatomy accurately in order to locate precisely the ablation lesions and minimize the complications. Currently, the most commonly used imaging method is that of multi-detector computed tomography (MDCT). Magnetic resonance imaging is used less commonly because of its lower spatial resolution, longer procedure and worse availability (1). The goal of MDCT examination is to create a 3D digital fusion of MDCT data with a fast electroanatomic map (FAM). It is also used for visual inspection of the anatomy when planning the intervention, e.g. when considering the use of balloon cryo-ablation. The use of MDCT images maximizes efficacy, shortens duration and generally makes CA more accurate (2-4). Despite the general agreement on the usefulness of MDCT, there is no consensus on specific technical parameters of MDCT acquisition. One of the controversial questions is whether ECG gat- 
Tab. 1. Demographic comparison of study groups.

\begin{tabular}{lccc}
\hline & $\begin{array}{c}\text { Gated } \\
\mathrm{n}=31\end{array}$ & $\begin{array}{c}\text { Non-Gated } \\
\mathrm{n}=31\end{array}$ & $\mathrm{p}$ \\
\hline Men & 22 & 21 & 1 \\
Women & 9 & 10 & 1 \\
age & $57.78 \pm 12.47$ & $55.65 \pm 12.27$ & 0.554 \\
BMI & $27.92 \pm 4.46$ & $29.66 \pm 3.51$ & 0.116 \\
\hline BMI - Body Mass Index, $\mathrm{p}-$ Level of significance
\end{tabular}

ing is absolutely necessary during MDCT data acquisition for CA purposes.

We have recently shown the impact of MDCT images with and without ECG gating on the data quality and radiation dose (5). The impact of different methods of CT data acquisition on the event-free survival rate after catheter ablation remains unknown.

\section{Material and methods}

\section{Study design}

The study is designed as a prospective, randomized, blinded study with a predefined MDCT and ablation protocol. Patients indicated for AF ablation were randomly divided into two equal groups by a radiologist blinded to their clinical status. Patients from group A underwent ECG-gated MDCT, while those from group B underwent helical nongated MDCT. Cardiac MDCT was performed 1-24 h before ablation. The study protocol was established prior to the enrollment of the first patient and applied in all aspects to all patients. The team performing AF ablation was blinded with respect to the MDCT technique used from enrollment until the end of the 12-month follow-up period after ablation.

\section{Patient group}

Patients with paroxysmal AF planned for CA were prospectively recruited.

Inclusion criteria: Paroxysmal AF, at least three episodes of $\mathrm{AF}$ in the last six months, age over 18 years, sinus rhythm during MDCT data acquisition, signed informed consent to this study.

Exclusion criteria: Persistent, long-standing or permanent AF, structural heart disease, valvular heart disease or history of valve disease surgery, use of amiodarone in the last three months, left ventricular ejection fraction $<35 \%$, pregnancy, breast feeding.

The study was approved by the Ethic Committee of the University Hospital Olomouc and Faculty of Medicine and Dentistry, Palacky University Olomouc. All patients signed an informed consent to participation in the study.

A total of 62 consecutive patients were enrolled and randomly divided into two groups as follows: group A (ECG-gated MDCT; $\mathrm{n}=31$ ) and group B (helical MDCT; no ECG synchronization; $\mathrm{n}$ = 31). There was no statistically significant difference in gender, age and body mass index (BMI) between the groups (Tab. 1).

\section{MDCT procedure}

All participants underwent examination on a 64-slice LightSpeed VCT scanner (General Electrics, Milwaukee, USA). The
ECG curve was monitored in all participants and all of them had a regular sinus rhythm during data acquisition. The examination ranged from the bifurcation of the trachea to the heart apex.

Patients from study group A underwent cardiac MDCT examination with retrospective ECG gating with an ECG-dependent tube current modulation as described previously (6).

Patients from study group B underwent helical MDCT without ECG gating. Both the ECG-gated and helical non-gated MDCT examinations were performed with anatomically adapted tube current modulation based on attenuation profiles in the antero-posterior and lateral directions. The participants in both groups received an intravenous dose of $70 \mathrm{~mL}$ of iodinated contrast agent Iopromide (Ultravist 370, Bayer Healthcare, Berlin, Germany) followed by a saline flush. The delay of data acquisition was triggered using the "bolus tracking" technique. More details on CT technique could be find in our previous work (5). The MDCT images were routinely evaluated by a radiologist, who also performed the scoring of the quality of visual data on a 5-point scale proposed by Wagner et al. as follows: 1 - excellent depiction of LA with smooth endocardial surface; 2 - good depiction of LA with only discrete irregularities of the endocardial surface; 3 - moderate depiction of LA with moderate irregularities of the endocardial surface; 4 poor depiction of LA with severe irregularities of the endocardial surface 5 - failed segmentation of LA (7).

\section{MDCT dosimetry}

The values of volume computed tomography dose index (CTDIvol) and dose length product (DLP) were recorded from the MDCT scanner dose report, which is calculated and archived automatically. Subsequently, simple effective dose (ED) estimation was calculated according to the European Guidelines on Quality Criteria European Guidelines for MDCT, namely by multiplying DLP by the region-specific normalized effective dose, which is $0.017 \mathrm{mSv}^{*} \mathrm{mGy}^{-1 *} \mathrm{~cm}^{-1}$ for chest CT (8).

\section{Catheter ablation}

The staff members performing the intervention were fully blinded with respect to which CT technique was used in a particular patient. The study protocol was established prior to the enrollment of the first patient and applied in all aspects to all patients. Ablation was performed under general anesthesia in all patients. Two sheaths were introduced via the femoral vein for an intracardiac echocardiography probe (AcuNav ultrasound catheter, Siemens Healthineers, USA) and decapolar diagnostic coronary sinus catheter (Inquiry ${ }^{\mathrm{TM}}$, St. Jude Medical, MN, USA). Two steerable transseptal sheaths (Agilis ${ }^{\mathrm{TM}}$ NxT Steerable Introducer, St. Jude Medical, MN, USA) were introduced via the femoral vein. A double transseptal puncture was performed in all patients. After the transseptal puncture, a 3D electroanatomic map was created using Carto3 ${ }^{\mathrm{TM}}$ (Biosense Webster, CA, USA). A fast anatomical map (FAM) was created consistently in all patients (Fig. 1). The anatomical map was merged with a 3D model of LA using the CartoMerge ${ }^{\mathrm{TM}}$ technology. A point-by-point radiofrequency wide antral ablation was performed in all patients using a Navistar ${ }^{\mathrm{TM}}$ ablation catheter (Biosense Webster, CA, USA) to 


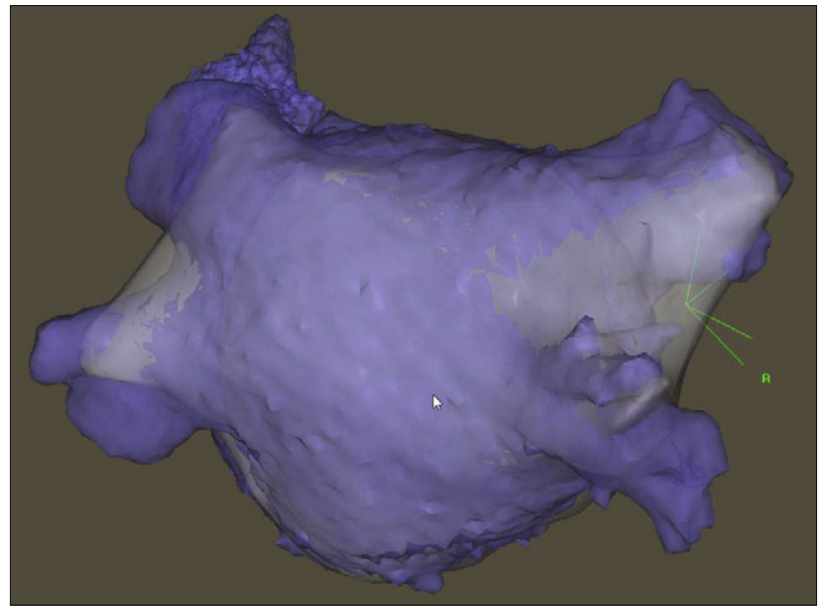

Fig. 1. Merge of left atrium maps: the blue map is the 3D MDCT model, the gray map is the electro-anatomic map.

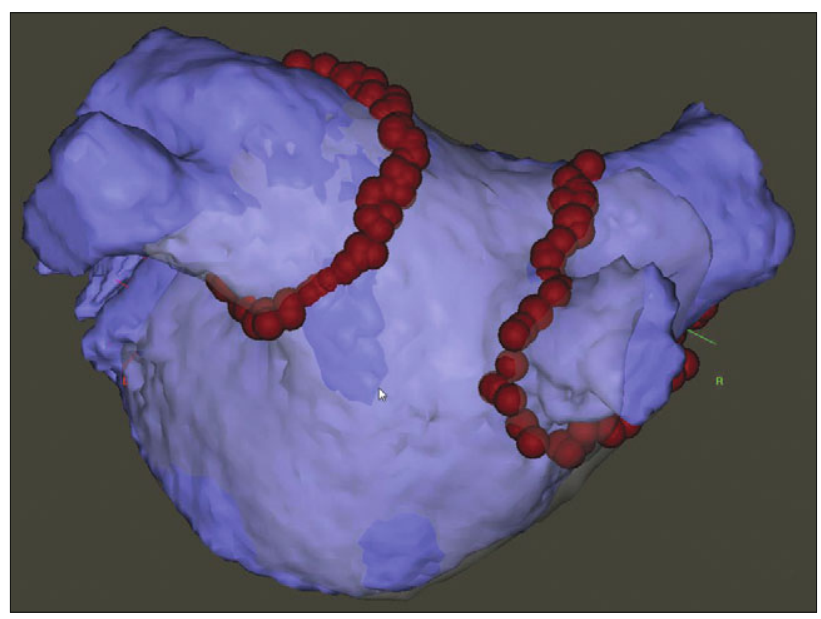

Fig. 2. CARTO3 CT map of the left atrium: red spots denote points of application of radiofrequency energy.

achieve isolation of the ipsilateral pulmonary veins (PVs) (Fig. 2). The ablation energy was set to $25-30 \mathrm{~W}$ in all patients with a cool flow of $20 \mathrm{~mL} / \mathrm{min}$. The inter-lesion distance was intended to be less than $6 \mathrm{~mm}$. A Lasso ${ }^{\mathrm{TM}}$ catheter (Biosense Webster, CA, USA) was used to validate the isolation of the PVs (entry block) in all patients. In the event of ongoing AF prior to the final check for the isolation of PVs, a direct current cardioversion was performed. Extrapulmonary ablation was not performed unless typical atrial flutter occurred or had been documented before ablation or if a spontaneous procedural atrial tachycardia occurred. Mapping and ablation of these cases of tachycardia were allowed. No inducibility testing or provocation of extra-pulmonary triggers was performed after the isolation of PVs. Thirty minutes after the isolation of PVs, adenosine was applied intravenously to test dormant conduction using a double lasso technique. An amount of at least $18 \mathrm{mg}$ of adenosine was used for the test. The dose was considered sufficient when it resulted in the second or third degree of atrioventricular block. Additional ablation was performed in patients with PVs with conduction recovery after the adenosine testing. To determine whether the dormant conduction was eliminated after reablation, another round of adenosine testing was performed. No specific waiting time was required before additional adenosine tests after reablation for reconduction. Once all the PVs with conduction recovery were re-isolated, the procedure was ended. The overall duration of the ablation procedure, fluoroscopy time and fluoroscopy dose were recorded at the end of the procedure. The automatic calculation of the registration error, i.e. the average distance between the respective points of the left atrium surface on the 3D MDCT model and FAM, was documented.

\section{Follow-up}

Four 7-day ambulatory ECG recordings were scheduled every 3 months (3, 6, 9, and 12 months after ablation) with a subsequent thorough clinical examination. The patients were instructed about the need of an early examination in the out-patient department in case of palpitations with an effort to document a possible arrhythmia in the period between 7-day ambulatory ECG monitoring procedures. Any documented arrhythmia (atrial flutter, atrial tachycardia, atrial fibrillation) lasting more than 30 seconds until the end of the FU was considered an arrhythmia recurrence. Blanking period was not used. Twelve months after ablation, all patients were divided according to the presence or absence of arrhythmia recurrence into two groups (A: AF-free, B: AF-recurrence).

\section{Statistics}

Parameters selected for statistical comparison between the groups were as follows: visual data quality, CTDIvol, DLP, effective dose of MDCT, mean registration error, duration of CA procedure, fluoroscopy time, dose during CA and rate of AF recurrence 12 months after ablation. Tests of normality (the Shapiro-Wilk test) revealed a non-normal distribution of data. The data were expressed as median, means and standard deviation (SD). The differences between the two independent groups were analyzed by means of the Fisher exact test and Mann-Whitney U-test. Differences in AF recurrence rate during follow-up period were compared using a chi-square test. Values with $\mathrm{p}<0.05$ were considered statistically significant. All statistical analyses were conducted with IBM SPSS Statistics 23 (IBM Corporation, 2015).

\section{Results}

All patients underwent the MDCT examination successfully. All examinations were appropriate for the subsequent ablation procedure.

\section{Visual quality of CT model of left atrium}

No statistically significant difference in the visual quality of MDCT images was found; in both groups the mean and median were around 2, i.e. discrete irregularities of the endocardial surface were found (Tab. 2a). 
177-183

\section{Dosimetry}

A comparison of dosimetric variables demonstrated the differences in CTDIvol, DLP and ED between the groups (mean values $89.6 \pm 6 \mathrm{mGy}, 1438.9 \pm 147.8 \mathrm{mGy} * \mathrm{~cm}, 24.46 \pm 2.51 \mathrm{mSv}$ in group A versus $19.2 \pm 4.3 \mathrm{mGy}, 328.2 \pm 73.8 \mathrm{mGy}^{*} \mathrm{~cm}, 5.58 \pm 1.26 \mathrm{mSv}$ in group B); these differences were statistically significant on the level of $\mathrm{p}<0.0001$ (Tab. 2a).

\section{Catheter ablation}

PV isolation (entry block) was achieved in all patients. The elimination of dormant conduction after the adenosine test was achieved in all PVs. There was no statistically significant difference between the groups in any of monitored parameters, namely duration of the procedure, fluoroscopy time, fluoroscopy dose and registration error (Tab. 2b).

\section{Atrial fibrillation recurrence after catheter ablation}

Twelve months after ablation, 25 of 31 and 24 of 31 patients in groups A and B, respectively, were free of AF (80.65 vs $77.42 \%$, $\mathrm{p}=0.838)($ Tab. 3).

\section{Discussion}

MDCT of LA and PVs represents a standard part of pre-procedure planning in many ablation centers because the fusion of $3 \mathrm{D}$ CT data and an electroanatomic map seems to maximize efficacy, shorten the duration and generally makes AF ablation more accurate (2-4). Having MDCT data available is certainly not absolutely necessary, especially when using intracardiac echocardiography (ICE). However, ICE is not used in most ablation centers and not all patients have good ICE image quality. In such cases, the 3D CT model can allow precise targeting of the ablation lesion. The 3D CT models of LA are used in many centers around the world and very likely are to be used in the future. Despite the general agreement on the usefulness of MDCT imaging, there is no broad consensus on the specific technical parameters of acquiring CT images. One of the questions remains unclear, namely whether ECG synchronization is absolutely necessary for CA. Since MDCT is a significant source of radiation, there is a strong effort to optimize the MDCT protocol so that the resulting MDCT images are of suf-

Tab. 2. Comparison of MDCT and catheter ablation parameters.

\begin{tabular}{lccc}
\hline MDCT parameters & Gated & Non-gated & $\mathrm{p}$ \\
\hline Visual quality & $1.77 \pm 0.88$ & $2.0 \pm 0.63$ & 0.102 \\
CTDIvol(mGy) & $89.55 \pm 5.99$ & $19.19 \pm 4.33$ & $<\mathbf{0 . 0 0 0 1}$ \\
DLP (mGy*cm) & $1438.87 \pm 147.75$ & $328.28 \pm 73.83$ & $<\mathbf{0 . 0 0 0 1}$ \\
Effective dose (mSv) & $24.46 \pm 2.51$ & $5.58 \pm 1.26$ & $<\mathbf{0 . 0 0 0 1}$ \\
\hline Catheter ablation parameters & Gated & Non-gated & $\mathrm{p}$ \\
\hline Registration error $(\mathrm{mm})$ & $2.42 \pm 0.72$ & $2.43 \pm 0.46$ & 0.612 \\
CA lenght (min) & $131.61 \pm 32.57$ & $119.84 \pm 33.18$ & 0.108 \\
Fluoroscopy time $(\mathrm{min})$ & $4.48 \pm 2.19$ & $3.89 \pm 1.83$ & 0.251 \\
Fluoroscopy dose $\left(\mathrm{Gy}^{*} \mathrm{~cm}^{2}\right)$ & $3.99 \pm 2.79$ & $3.91 \pm 2.91$ & 0.735 \\
\hline
\end{tabular}

$\mathrm{CTDI}_{\mathrm{vol}}$ - volume computed tomography dose index

DLP - dose lenght product

CA - catheter ablation
Tab.3. AF recurrence rate in both study groups

\begin{tabular}{lccc}
\hline & AF-free & AF recurrence & p \\
\hline Gated & $25(80.65 \%)$ & $6(19.35 \%)$ & 0.838 \\
Non-Gated & $24(77.42 \%)$ & $7(22.58 \%)$ & \\
\hline
\end{tabular}

$\mathrm{AF}$ - atrial fibrillation, $\mathrm{p}$ - significance level

ficient quality and the radiation dose is the lowest possible. The endeavor to reduce the radiation dose is very important as another dose of radiation is applied to the patient because of fluoroscopy during CA; furthermore, in the event of a recurrence of AF, a new MDCT is often obtained. Another MDCT is sometimes used to assess complications after CA.

One of the aspects affecting MDCT image quality and radiation dose is the use of synchronization of data acquisition with an ECG curve in the MDCT scanner. The main technical difference affecting the radiation dose between ECG-gated MDCT and helical non-gated MDCT is the value of the pitch, which is defined as the distance that the table travels per complete rotation of the gantry divided by the detector width. For helical MDCT scanning, a higher pitch can be used, whereas for ECG-gated MDCT a lower pitch is necessary in order to provide partially overlapping MDCT projections that allow the data from different phases of the cardiac cycle to be sorted (9). Thus, the pitch is inversely proportional to the resulting radiation dose. It is absolutely essential to use ECG gating to perform CT coronarography, but the question is whether this approach is also unavoidable in the MDCT of LA and PVs. Besides the retrospective ECG gating, it is also possible to use prospective ECG triggering (also known as the "step-and-shoot" technique), in which the data acquisition is limited only to the diastole, thus reducing the radiation dose, whereas other phases of the cardiac circle are not available for post-processing (10). Both prospectively ECG-triggered and retrospectively ECG-gated techniques can be compromised by the presence of stair-step artifacts significantly reducing the image quality of the MDCT model ( 9 , $11,12)$. In our work, we strictly used retrospective ECG gating.

The arguments for ECG synchronization include fewer motion artifacts of LA contours and ostia of PVs, possibility of observing the movement of LA wall and changes in diameter of PVs during the heart cycle, or choice to select the optimal phase of the heart cycle for the subsequent fusion.

The argument against ECG synchronization is that non-synchronized MDCT images may be blurred as a result of motion, and yet they are fully usable for both anatomical visual evaluation and subsequent digital fusion. Furthermore, it is possible to question the importance of displaying the LA volume and changes in the diameter of pulmonary veins. These changes are measurable, but they are likely to be negligible with regard to the ablation performance because ablation systems do not allow ablation to be limited to a particular phase of the heart cycle and therefore a certain inaccuracy is always present even when using best-quality ECG-gated MDCT data. So far, two published papers compared directly the image quality and radiation dose of ECG-gated and helical nongated MDCT protocols. However, both papers were retrospective, while one of them included only a limited number of subjects and, 

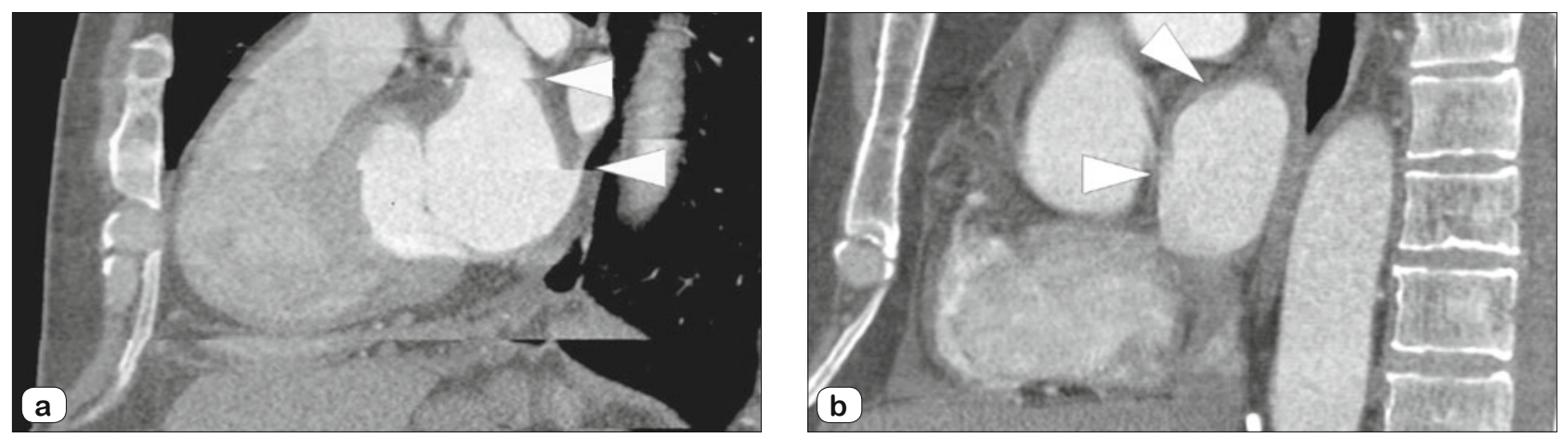

Fig. 3. a) Retrospectively ECG-gated MDCT in the sagittal plane; the images show stair-step artifacts on the surface of the left atrium (arrowheads) resulting from incorrect data registration; the visual image quality was graded as "poor"; b) Helical non-gated MDCT in the sagittal plane; the images show blurred surface of the left atrium (arrowheads) as a result of motion; the visual image quality was graded as "moderate".

in particular, neither of these two papers also evaluated the effect of the MDCT technique on performance, parameters and results of subsequent CA $(7,10)$.

The aims of our study were to provide a prospective, randomized, blinded comparison of MDCT image quality between the ECG-gated and helical non-gated techniques, comparison of the radiation dose between the two methods, and, since most centers prefer the MDCT of LA using ECG gating, an evaluation of the effect of MDCT data quality on the ablation process (13-19).

The main argument for the use of ECG gating is that of a better visual quality of the LA MDCT model. The results of our work are in disagreement with this premise. In group A (ECG-gated MDCT), the average visual quality score was 1.77 points out of five. Low-score ECG-gated images (3 or 4 points) were compromised by stair-step artifacts as a result of cardiac motion or patients' non-compliance with the instruction to hold their breath (Fig. 3a). In group B (helical non-gated MDCT), the average visual quality score was two points out of five. Five patients showed blurred LA contours as a result of motion and were rated as "moderate” (Fig. 3b). Thus, our work does not confirm the assumption that ECGgated MDCT data possess a better visual quality. The comparable visual quality of the data between the two techniques was previously published by other teams $(7,10)$. Moreover, the blurring of LA contours is closer to the real-world reality of a beating heart than the sharp stair-step artifact.

The evaluation of the visual quality of CT images is subjective. Therefore, in addition to visual quality, we also used an objective measurement of a match between the 3D MDCT model surface and FAM of LA. This deviation (also called a registration error), can be expressed simply as the mean distance between the corresponding points in the CT model and FAM measured in millimeters. We did not find a statistically significant difference between the mean registration error between groups A and B (2.42 mm vs $2.43 \mathrm{~mm}$ ), which is also consistent with previous research (7). In other words, for the physician performing the intervention, the question as to which MDCT technique is to be used is completely insignificant; the fit in the 3D MDCT model and FAM was the same.

Another crucial aim of our study was to compare the radiation dose between ECG-gated and helical non-gated MDCT. The values of CTDIvol and DPL were chosen for the comparison. From the aspect of MDCT technique, the two variables are more suitable for comparing different MDCT protocols than ED because they are better-defined quantities and their calculation is not influenced by the selected methodology. For both CTDIvol and DPL, a significantly higher value was found for ECG-gated MDCT. Both values were about 4.5 times higher, which is directly projected into the subsequently calculated ED estimations. The cause of this multiple difference lies undoubtedly in the different values of pitch, which are inversely proportional to the resulting CTDIvol and DLP. The difference in pitch values for ECG-gated MDCT (0.2:1) and helical non-gated MDCT (0.984:1) apparently inversely approximates the difference between the average values of CTDIvol and DLP. Our DLP and ED values of the helical non-gated MDCT were comparable to those reported in the previous article (7); for ECG-gated MDCT, the mean values were relatively higher, probably because we used a protocol originally intended for CT coronarography. If, however, we compare the observed ED values with other papers using 64-row scanners for retrospectively ECG-gated cardiac MDCT, the ED values are comparable (20). The comparison of ED between different studies may be complicated because it is only an estimation, which may vary considerably according to the methodology or software used. The easiest way to estimate ED is to multiply DLP by an organ-specific coefficient. We used the commonly used chest CT coefficient, i.e. $0.017 \mathrm{mSv}^{*} \mathrm{mGy}^{-1 *} \mathrm{~cm}^{-1}$ $(8,21)$, but there are also different recommendations for chest CT, e.g. $0.019 \mathrm{mSv}^{*} \mathrm{mGy}^{-1 *} \mathrm{~cm}^{-1}$ (22). Additionally, there are works recommending a coefficient of $0.028 \mathrm{mSv}^{*} \mathrm{mGy}^{-1 *} \mathrm{~cm}-1$ for cardiac MDCT (23); in this respect, the ED values can be systematically underestimated.

Besides the pitch value, the radiation dose of MDCT may also be affected by other parameters. Therefore, it is necessary to find a suitable compromise between the technical quality of MDCT images and the resulting radiation dose. In addition to, e.g., slice thickness, anatomically adapted tube current modulation or craniocaudal extent of the examination, the modern MDCT scanners offer modern image reconstruction algorithms. Compared to the traditionally used filtered back-projection technique, contemporary scanners have the option of iterative reconstruction algorithms such 
as adaptive statistical iterative reconstruction. These techniques reduce the noise in CT image and thus allow reduction of voltage and current values of the X-ray tube. In recent years, more advanced iterative techniques (e.g. model-based iterative reconstruction) have become available; their long calculation time is a disadvantage, but they can reduce the ED to below $1 \mathrm{mSv}$ (24). ECG-gated scan dose reduction can be also achieved by using ECG-controlled tube current modulation technique (also referred to as "ECG pulsing"). This algorithm modulates tube current according to cardiac cycle phases. As the cardiac motion is greatest during systole and least during diastole, the image data are most likely sharpest during diastole. Accordingly, this algorithm reduces the tube current by $75 \%$ during systole, in which image reconstruction is not likely to be of interest (6).

Complete isolation of PVs was achieved in all patients. There was no positive effect of ECG gating on the duration of ablation procedure; the duration of CA was even 14 minutes shorter in patients with helical non-gated MDCT, but this difference was not statistically significant. A similar situation occurred in the comparison of fluoroscopy time during CA. In group B, the fluoroscopy time was insignificantly shorter than that in group A. Therefore, our research did not demonstrate any positive effect of ECG gating on the performance and immediate result of CA.

We have prospectively observed the whole patient group with four 7-day ambulatory ECG monitoring cycles. Twelve months after CA, the whole follow-up period was evaluated in all patients. There was no difference in the CA outcome. Since a really good correlation of anatomical map and 3D CT model was documented in both subgroups, a significant difference between both subgroups would be surprising. Nevertheless, no paper has been published so far with a prospective randomized comparison of gated and non-gated CT acquisition of LA before AF catheter ablation with a precise evaluation of CA outcome. In our study, a helical non-gated MDCT achieved a radiation dose more than four times lower than ECG-gated MDCT with absolutely no impact on CA event-free survival date. Currently, there is a general agreement on the usefulness of MDCT but there is no consensus on specific technical parameters of MDCT acquisition. Based on our results, there is no reason to use ECG-gating in these patients.

\section{Study limitations}

Our study has several limitations. Compared to previously published studies, the prospective character of the study, with standard randomization and double blindness, is among the merits of our work. Randomization was performed by a radiologist blinded to the patients' health conditions. The entire intervention team remained blinded to MDCT technique used. Furthermore, our study group was larger than that of Weber et al. (7) and more homogeneous than the group of Thai et al. At the same time, it also exceeds the number of subjects in several subgroups (10). It also contains a direct comparison of the effects of two MDCT techniques on subsequent CA and its outcome. It must be admitted however, that a disadvantage might lie in the fact that the trend for research in cardiac imaging is currently towards 320-row or dual-source scanners. Nevertheless, 64-row and 128-row scanners, which offer a choice between ECG-gated and helical non-gated techniques, are still widely used for CA planning in clinical practice. The choice between high-pitch helical technology and ECG-triggering/gating is also relevant in dual-source MDCT scanners $(10,25)$. Thus, our research is less relevant only to 320-row MDCT scanners with volume data acquisition.

Limitations may include the use of a protocol originally designed for CT coronarography, where much greater detail is needed than with LA imaging. It would be possible to find better compromise between signal-to-noise value, spatial resolution and radiation dose for LA imaging in clinical practice. Theoretically, it might be more appropriate to use prospective ECG triggering, as it is likely that prospective triggering would achieve a lower ED than retrospective gating. However, the main goal of our study was to confirm that regardless which ECG synchronization technique it is compared to, the helical non-gated MDCT is a fully adequate method with comparable image quality and CA outcome.

\section{Conclusion}

When compared to ECG-gated multi-detector computed tomography, the helical non-gated multi-detector computed tomography of the left atrium and pulmonary veins achieved a radiation dose more than four times lower with comparable visual image quality, registration error between 3D MDCT model and anatomical map, and event-free survival rate after catheter ablation.

\section{References}

1. Hamdan A, Charalampos K, Roettgen R, Wellnhofer E, Gebker R, Paetsch I, Jahnke C, Schnackenburg B, Tang M, Gerds-Li H, Fleck E. Magnetic Resonance Imaging Versus Computed Tomography for Characterization of Pulmonary Vein Morphology Before Radiofrequency Catheter Ablation of Atrial Fibrillation. Am J Cardiol 2009; 104 (11): 1540-1546.

2. Bertaglia E, Bella PD, Tondo C, Proclemer A, Bottoni N, De Ponti R, Landolina M, Bongiorni MG, Coro L, Stabile G, Russo AD, Verlato R, Mantica M, Zoppo F. Image integration increases efficacy of paroxysmal atrial fibrillation catheter ablation: results from the CartoMerge Italian Registry. EP Eur 2009; 11 (8): 1004-1010.

3. Della Bella P, Fassini G, Cireddu M, Riva S, Carbucicchio C, Giraldi F, Maccabelli G, Trevisi N, Moltrasio M, Pepi M, Galli CA, Andreini D, Ballerini G, Pontone G. Image integration-guided catheter ablation of atrial fibrillation: a prospective randomized study. J Cardiovasc Electrophysiol 2009; 20 (3): 258-265.

4. Martinek M, Nesser H-J, Aichinger J, Boehm G, Purerfellner H. Impact of integration of multislice computed tomography imaging into three-dimensional electroanatomic mapping on clinical outcomes, safety, and efficacy using radiofrequency ablation for atrial fibrillation. Pacing Clin Electrophysiol PACE 2007; 30 (10): 1215-1223.

5. Tudos $\mathbf{Z}$ et al. ECG non-gated multi-detector computed tomography protocol prior to catheter ablation of atrial fibrillation provides sufficient data quality with lower radiation exposure compared to ECG-gated protocol-results of a prospective, randomized and blinded study. 2018. 
6. Hermann F, Martinoff S, Meyer T, Hadamitzky M, Jiang C, Hendrich E, Hausleiter J. Reduction of radiation dose estimates in cardiac 64-slice CT angiography in patients after coronary artery bypass graft surgery. Invest Radiol 2008; 43 (4): 253-260.

7. Wagner M, Butler C, Rief M, Beling M, Durmus T, Huppertz A, Voigt A, Baumann G, Hamm B, Lembcke A, Vogtmann T. Comparison of non-gated vs. electrocardiogram-gated 64-detector-row computed tomography for integrated electroanatomic mapping in patients undergoing pulmonary vein isolation. Europace 2010; 12 (8): 1090-1097.

8. Bongartz G, Golding SJ, Jurik AG, Leonardi M, van Persijn van Meerten E, Geleijns J, Jessen K., Panzer W, Shrimpton PC, Tosi G. European Guidelines on Quality CriteriaEuropean Guidelines for Multislice Computed Tomography. 1999. www.drs.dk/guidelines/ct/quality/ mainindex.htm>.

9. Desjardins B, Kazerooni EA. ECG-Gated Cardiac CT. Am J Roentgenol 2004; 182 (4): 993-1010.

10. Thai W, Wai B, Lin K, Cheng T, Heist EK, Hoffmann U, Singh JP, Truong QA. Pulmonary Venous Anatomy Imaging With Low-Dose, Prospectively ECG-Triggered, High-Pitch 128-Slice Dual-Source Computed TomographyClinical Perspective. Circ Arrhythm Electrophysiol 2012; 5 (3): 521-530.

11. Husmann L, Valenta I, Gaemperli O, Adda O, Treyer V, Wyss CA, Veit-Haibach P, Tatsugami F, Schulthess V, K G, Kaufmann PA. Feasibility of low-dose coronary CT angiography: first experience with prospective ECG-gating. Eur Heart J 2008; 29 (2): 191-197.

12. Machida H, Tanaka I, Fukui R, Shen Y, Ishikawa T, Tate E, Ueno E. Current and Novel Imaging Techniques in Coronary CT. RadioGraphics 2015; 35 (4): 991-1010.

13. Cronin P, Kelly AM, Desjardins B, Patel S, Gross BH, Kazerooni EA, Morady F, Oral H, Carlos RC. Normative Analysis of Pulmonary Vein Drainage Patterns on Multidetector CT With Measurements of Pulmonary Vein Ostial Diameter and Distance to First Bifurcation. Acad Radiol 2007; 14 (2): 178-188.

14. Manghat NE, Mathias HC, Kakani N, Hamilton MCK, MorganHughes G, Roobottom CA. Pulmonary venous evaluation using electrocardiogram-gated 64-detector row cardiac CT. Br J Radiol 2012; 85 (1015): 965-971.

15. Choi SI, Seo JB, Choi SH, Lee S-H, Do K-H, Ko SM, Lee JS, Song J-W, Song K-S, Choi K-J, Kim Y-H, Lim T-H. Variation of the size of pulmonary venous ostia during the cardiac cycle: optimal reconstruction window at ECG-gated multi-detector row CT. Eur Radiol 2005; 15 (7): 1441-1445.
16. Avelar E, Durst R, Rosito GA, Thangaroopan M, Kumar S, Tournoux F, Chan RC, Hung J, Hoffmann U, Abbara S, Brady T, Cury RC. Comparison of the accuracy of multidetector computed tomography versus two-dimensional echocardiography to measure left atrial volume. Am J Cardiol 2010; 106 (1): 104-109.

17. Pontone G, Andreini D, Bertella E, Petullà M, Russo E, Innocenti E, Mushtaq S, Gripari P, Loguercio M, Segurini C, Baggiano A, Conte E, Beltrama V, Annoni A, Formenti A, Guaricci AI, Casella M, Fassini G, Giovannardi M, Veglia F, Tondo C, Pepi M. Comparison of cardiac computed tomography versus cardiac magnetic resonance for characterization of left atrium anatomy before radiofrequency catheter ablation of atrial fibrillation. Int J Cardiol 2015; 179: 114-121.

18. Neumann A, Bohata S, Kerkovsky M, Pavlik T. Effect of the presence of calcium in the coronary arteries to the cardiac function. Ceska Radiol 2011; 65 (4): 279-288.

19. Fahlenkamp UL, Lembcke A, Roesler R, Schwenke C, Huppertz A, Streitparth F, Taupitz M, Hamm B, Wagner M. ECG-gated imaging of the left atrium and pulmonary veins: Intra-individual comparison of CTA and MRA. Clin Radiol 2013; 68 (10): 1059-1064.

20. Sun Z. Coronary CT angiography with prospective ECG-triggering: an effective alternative to invasive coronary angiography. Cardiovasc Diagn Ther 2012; 2 (1): 28-37.

21. Huda W, Ogden KM, Khorasani MR. Converting Dose-Length Product to Effective Dose at CT. Radiology 2008; 248 (3): 995-1003.

22. Bongartz G, Golding SJ, Jurik AG, Leonardi M, van Persijn van Meerten E, Rodriguez R, Schneider K, Calzado A, Geleijns J, Jessen K., Panzer W, Shrimpton PC, Tosi G. European Guidelines for Multislice Computed Tomography. European Commission (2004).

23. Gosling O, Loader R, Venables P, Rowles N, Morgan-Hughes G, Roobottom C. Cardiac CT: are we underestimating the dose? A radiation dose study utilizing the 2007 ICRP tissue weighting factors and a cardiac specific scan volume. Clin Radiol 2010; 65 (12): 1013-1037.

24. Annoni AD, Andreini D, Pontone G, Formenti A, Petullà M, Consiglio E, Nobili E, Baggiano A, Conte E, Mushtaq S, Bertella E, Billi F, Bartorelli AL, Montorsi P, Pepi M. Ultra-low-dose CT for left atrium and pulmonary veins imaging using new model-based iterative reconstruction algorithm. Eur Heart J Cardiovasc Imaging 2015; 16 (12): 1366-1373.

25. Iwayama T, Arimoto T, Ishigaki D, Hashimoto N, Kumagai YU, Koyama YO, Kiribayashi N, Netsu S, Nishiyama S, Takahashi H, Shishido T, Miyamoto T, Sato T, Watanabe T, Kubota I. The Clinical Value of Nongated Dual-Source Computed Tomography in Atrial Fibrillation Catheter Ablation. J Cardiovasc Electrophysiol 2016; 27 (1): 34-40. 